\title{
An Eye on Breast and Prostate Cancers
}

\author{
Shalini Gandi ${ }^{*}$, Phaneendra $\mathrm{M}^{2}$, Anamika $\mathrm{K}^{3}$ and Jyothi $\mathrm{J}^{4}$ \\ ${ }^{1}$ Department of Biochemistry, Andhra University, Andhra Pradesh, India \\ ${ }^{2}$ Department of Biotechnology, GITAM University, Andhra Pradesh, India \\ ${ }^{3}$ Department of Industrial Biotechnology, Berhampur University, Odisha, India \\ ${ }^{4}$ Department of Biotechnology, Osmania University, Andhra Pradesh, India
}

\begin{abstract}
Cancer is a class of diseases characterized by out-of-control cell growth. There are over 100 different types of cancer, and each is classified by the type of cell that is initially affected. In all these types of cancers breast and prostate cancers are the most prevalent and dangerous. In this review article a field of vision is given on these two hazardous cancers.
\end{abstract}

Keywords: Breast cancer; Prostate cancer; Cancer therapy; Biomarkers; Vacuum-assisted breast biopsy; Prostate specific antigen testing

\section{Introduction}

Cancer is a major public health problem in the United States and many other parts of the world. Currently, one in four deaths in the United States is due to cancer [1]. Breast cancer and prostate cancer are the two most common invasive cancers in women and men, respectively. Breast cancer is one of the most frequently diagnosed cancers among women, with an estimated 184,450 new cases and 40,930 deaths in 2008 [2]. Prostate cancer (PC) is the second leading cause of cancer deaths in men in America and Western Europe [3]. Prostate cancer is most frequently diagnosed among men, with an estimated 192,280 and 27,360 deaths in 2009 [1]. Although these cancers arise in organs that are different in terms of anatomy and physiological function both organs require gonadal steroids for their development, and tumors that arise from them are typically hormone-dependent and have remarkable underlying biological similarities [4].

\section{Risk factors}

Genetic: Recent rapid increases in our understanding of the genetic basis of breast cancer have created a resurgent interest in identifying those at increased risk because of family history. The risk of breast cancer associated with a family history is higher in younger women, some studies suggest that the risk is particularly high in women under 40 and then falls off rapidly to a background level of elevated risk. Nine studies reported the risk of breast cancer in women whose daughter had had the disease [5]. A case-control study was performed to estimate the relative risk of developing prostate cancer for men with a positive family history. Prostate cancer had a higher degree of familiality than either breast or colon cancer two cancers with well-recognized familial tendencies. In a recent study involving 691 prostate cancer cases and the male relatives of their wives, an increased risk of prostate cancer among men was observed whose first or second degree relatives also had prostate cancer [6].

Hormonal: Breast cancer is a steroid hormone-dependent tumor. Many studies have reported a correlation between elevated estrogen blood levels and breast cancer and this observation has raised controversy concerning the long-term use of hormonal replacement therapy [7]. Stratification of patients according to hormone (ER/ PR) receptor status and nodal metastasis is of great therapeutic importance [8]. Several years ago, only age, race, and family history were known as risk factors for this diseases. However, today, much progress has been made towards discovering hormonal risk factors for prostate cancer [9]. Prostate cancer is a hormone-dependent, epithelial-derived tumor, resulting from uncontrolled growth of genetically unstable transformed cells [10]. High androgen receptor (AR) level in primary tumor predicts increased prostate cancer-specific mortality. However, the mechanisms that regulate AR function in prostate cancer are poorly known [11]

\section{Dietary}

High-energy intake in relation to physical activity, which accelerates growth and the onset of menstruation during childhood, leads to weight gain in middle life and thus can contribute substantially to breast cancer risk. Some evidence suggests that vitamin A or other compounds in vitamin A-rich foods may reduce breast cancer risk modestly, but these findings are not conclusive and deserve further consideration [12]. Breast cancer incidence and mortality rates decrease with environmental conditions that promote Vitamin D synthesis in human skin including lower latitude and higher personal exposure to sunlight [13].

While a number of dietary factors have been linked to prostate cancer the evidence is still tentative [14]. Evidence supports little role for dietary fruits and vegetables in prostate cancer occurrence [15]. Red meat and processed meat also appear to have little effect [16]. Lower blood levels of vitamin D may increase the risk of developing prostate cancer [17]. This may be linked to lower exposure to ultraviolet (UV) light, since UV light exposure can increase vitamin D in the body [18].

\section{Medical exposure}

Moderate to high-dose radiotherapy is known to increase the risk of breast cancer. Uncertainties remain about the effects of low-dose chest

*Corresponding author: Shalini Gandi, Department of Biochemistry, Andhra University, Visakhapatnam, Andhra Pradesh, India, E-mail: shalini.msc28@ gmail.com

Received August 02, 2011; Accepted September 30, 2011; Published October 02, 2011

Citation: Shalini G, Phaneendra M, Anamika K, Jyothi J (2011) An Eye on Breast and Prostate Cancers. J Cancer Sci Ther S17. doi:10.4172/1948-5956.S17-003

Copyright: @ 2011 Shalini G, et al. This is an open-access article distributed under the terms of the Creative Commons Attribution License, which permits unrestricted use, distribution, and reproduction in any medium, provided the original author and source are credited. 
X-rays, particularly in individuals at increased genetic risk [19]. There are also some links between prostate cancer and medications, medical procedures, and medical conditions [20]. Use of the cholesterollowering drugs known as the statins may also decrease prostate cancer risk [21].

\section{Viral}

Many viruses have been associated with human breast cancers, including Epstein-Barr and Cytomegalovirus. New evidence has revealed the frequent presence of high risk human papillomavirus (HPV) strains HPV16 and HPV18 in breast carcinoma biopsies [22]. In 2006, researchers associated a previously unknown retrovirus, Xenotropic MuLV-related virus or XMRV, with human prostate tumors [23]. Subsequent reports on the virus have been contradictory. A group of US researchers found XMRV protein expression in human prostate tumors [24].

\section{Racial differences}

In case of breast cancer the hypothesis that white women with breast cancer survive longer than black women with this disease was evaluated in a retrospective analysis of a population-based prospective follow-up study. The racial difference in survival was greatest among women with advanced disease, and a higher proportion of black women with advanced disease did not receive surgery. Even when the type of surgery and stage of disease were controlled, race was a significant prognostic factor [25].

In case of prostate cancer, black men represent a particularly high risk group for the development of prostate cancer. But little is known of the molecular genetic factors, tumor suppressor genes and/or oncogenes that play a role in prostate cancer in black men. Increased research efforts are needed in order to understand this problem at the molecular level [26].

\section{Metabolic syndrome and breast cancer}

Metabolic syndrome components are hypertension, dyslipidemia, glucose intolerance and obesity. Metabolic syndrome, also called insulin resistance syndrome, is defined as a constellation of metabolic abnormalities, including glucose intolerance (fasting plasma concentrations _ $110 \mathrm{mg} / \mathrm{dL}$ ), dyslipidemia (serum triacylglycerol concentration _ $150 \mathrm{mg} / \mathrm{dL}$, serum HDL concentration _ $40 \mathrm{mg} / \mathrm{dL}$ ), hypertension (blood pressure_130/85 $\mathrm{mm} \mathrm{Hg}$ ), and obesity (a waist circumference of _102 cm) [27]. In addition to steroid hormones, polypeptide growth hormones (insulin-like factors and insulin itself) are thought to play an important role in regulation of breast tumor proliferation [28].

Hypothesis was raised that breast cancer risk is determined by cell proliferation in response to sex hormones, but also to polypeptide growth hormones. Clinical and epidemiological evidence suggests that both breast cancer and the metabolic disorders included in the insulin resistance syndrome are of polygenic and multifactorial origin. Experimental evidence suggests that hyperinsulinemia and its features can increase the promotion of mammary carcinogenesis and the mechanism is likely related to increased bioactivity of insulin-like growth factor 1 (IGF-1) [29].

Some findings are consistent with insulin-stimulate growth promotion at supraphysiologic insulin concentrations (as happens in metabolic syndrome) mediated through the IGF-1 and insulin receptors. There is a possibility that effect of BMI on breast cancer risk is mediated by both insulin resistance and estrogen metabolism 4 .

In our study, obesity and upper body fat distribution are not related to the breast cancer probably because our subjects were affected by breast cancer at various degrees of pathology [30].

A family history of breast cancer is quite common in the general population, but preventive interventions targeted to women at risk of breast cancer because of family history will have limited impact on breast cancer morbidity as a whole. Another study evidences the association between physical activity and breast cancer only for postmenopausal women. So, nutrition and lifestyle modifications that improve insulin sensitivity may not only decrease a tendency to atherosclerosis but also reduce breast cancer risk in women. Weight reduction combined with a program of regular physical exercise has been shown to reduce both estrogen and insulin concentrations in obese women and such a regimen might be tested in clinical trials for an effect on breast cancer risk in obese women. Interventions to decrease breast cancer risk in first-degree relatives of breast cancer patients need to begin at an early age. The prevalence of type 2 diabetes mellitus, hypertension, dyslipidemia, was higher in group of women affected by breast cancer vs. benign breast pathology and controls. The findings are in an agreement with the hypothesis of the interrelationship of hyperinsulinism/insulin resistance with the growth-related abnormalities of breast cancer [31].

\section{Metabolic syndrome and prostate cancer}

Studies indicate that metabolic syndrome is associated with prostate cancer. A systematic literature search for studies on metabolic syndrome or insulin resistance and prostate cancer was performed using the OVID database manager searching the Medline and EMBASE databases. Seven studies were included, of which five were prospective cohorts and two were case-control studies. Most other studies were reviews. Four studies showed a positive association, one showed a negative association and one showed no association. One study, using two different analyses for metabolic syndrome, showed a positive association, or none, depending on the criteria used for defining metabolic syndrome. The results indicate that metabolic syndrome can be used to identify men at risk of prostate cancer. The definition of the metabolic syndrome must be taken into consideration. Despite the strong association of diabetes with obesity and insulin resistance, diabetes has been consistently linked to a reduced risk of prostate cancer in several prospective and case control studies, although the magnitude of the association is modest.

Several mechanisms could explain the association of obesity and metabolic syndrome with prostate cancer risk, including the sex steroid hormone, insulin and IGF signaling, and inflammation pathways.

Data on metabolic syndrome and prostate cancer are limited, and few studies have studied all 4 components (hypertension, dyslipidemia, glucose intolerance and obesity) of metabolic syndrome. It is unclear whether a change in one or 2 components can reflect the overall effect of metabolic syndrome. Thus, we conclude that although the metabolic syndrome hypothesis is highly plausible, currently, the epidemiologic evidence is insufficient to suggest a link between metabolic syndrome and prostate cancer [27].

\section{Diagnosis}

The first cancer screening test to be widely used was the Pap test. The test was first developed by George Papanicolaou as a method in understanding the menstrual cycle. He also identified Pap tests 
potential for early detection of cervical cancer. In 1960s mammography was developed for identification of breast cancer. Later early detection of cervix, breast, colon, rectum, endometrium, prostate, thyroid, oral cavity, skin, lymph nodes, testes, and ovaries cancers were identified and practiced in the clinic [32].

The earliest breast cancers are detected by a mammogram. Mammography and clinical breast exam are used for screening breast cancer. This indicates an approximate likelihood that the lump is cancer, and may also detect some other lesions [33]. When the tests are inconclusive Fine Needle Aspiration and Cytology (FNAC) may be used. FNAC may be done in a GP's office using local anaesthetic if required, involves attempting to extract a small portion of fluid from the lump. Clear fluid makes the lump highly unlikely to be cancerous, but bloody fluid may be sent off for inspection under a microscope for cancerous cells. Together, these three tools can be used to diagnose breast cancer with a good degree of accuracy. Other options for biopsy include core biopsy, where a section of the breast lump is removed, and an excisional biopsy, where the entire lump is removed. In addition vacuum-assisted breast biopsy (VAB) may help diagnose breast cancer among patients with a mammographically detected breast in women [34]. A data suggest that c-erbB-2 and $\mathrm{p}^{53}$ expression in breast cancer have prognostic significance [35]. It has been demonstrated that the tumor angiogenic ability is one of the most important predictors of breast cancer progression. Factors controlling tumor angiogenesis are varied and currently under investigation. Chemokines, which produced by both tumor cells and cells of tumor microenvironment are known with a role in tumor angiogenesis [36]. Elastic-scattering spectroscopic techniques are also used for the diagnosis of breast cancer for preliminary clinical results [37].

The last 20 years have seen remarkable changes in both the diagnosis and treatment of prostate cancer. Two driving forces have been the development of prostate-specific antigen (PSA) testing as well as the availability of effective pharmacologic interventions to reduce or eliminate the effects of androgens. In case of prostate cancer, for early diagnosis Prostate Specific Antigen testing (PSA testing) can be done. This test gives a better short term outcomes after diagnosis [38]. Ultrasound (US) and Magnetic Resonance Imaging (MRI) are the two main imaging methods used for prostate cancer detection. If cancer is suspected, a biopsy is offered expediently. During a biopsy a urologist or radiologist obtains tissue samples from the prostate via the rectum. A biopsy gun inserts and removes special hollow-core needles (usually three to six on each side of the prostate) in less than a second. Prostate biopsies are routinely done on an outpatient basis and rarely require hospitalization. Fifty-five percent of men report discomfort during prostate biopsy [39].

Now a day's molecular biomarkers are playing key role in diagnosis of both breast and prostate cancer. A biomarker can be a substance that is introduced into an organism as a means to examine organ function or other aspects of health. There are different types of biomarkers like

- $\quad$ Protein biomarkers

- Molecular biomarkers

- Food biomarkers

- $\quad$ Blood biomarkers

- Urinary biomarkers

- $\quad$ Tissue biomarkers
- Clinical biomarkers

- Prognostic biomarkers

- Predictive biomarkers

- Biomarkers for early detection

- Biomarkers based on cancer types

The process of the detection methods had made simple yet somewhat complicated though according to a review [40].

\section{General mechanism of cancer development}

Cell division is a physiological process that occurs in almost all tissues and under many circumstances. Under normal circumstances, the balance between proliferation and programmed cell death, usually in the form of apoptosis, to maintain the integrity of organs and tissues. Certain mutations in DNA tumor suprresor genes get deactivated, lead to cancer, disrupting the orderly physiological processes (Figure 1).

Mutations that can lead to breast cancer have been experimentally linked to estrogen exposure [41]. These mutations are either inherited or acquired after birth. Normal cells divide as many times as needed and stop. They attach to other cells and stay in a place in tissues. This is benign breast disease [42]. These cells become cancerous when mutations destroy their ability to stop dividing, to attach to other cells and to stay where they belong. A prospective study of benign breast disease and the risk of breast cancer confirm the remarked increase in

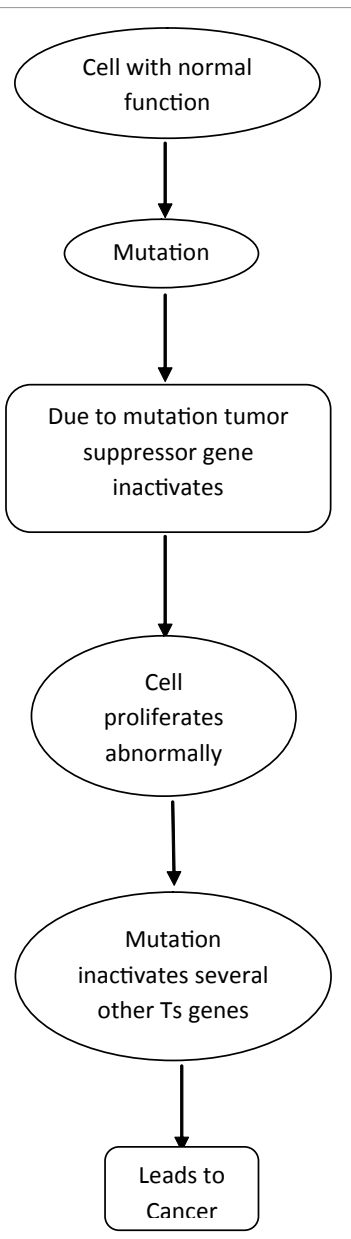

Figure 1: General mechanism of cancer development. 
breast cancer risk among women with atypical hyperplasia, particularly in premenopausal women, and suggest that these women should be encouraged to undergo frequent breast cancer screening [43]. Presumably, they allow the other mutations, which allow uncontrolled division, lack of attachment, and metastasis to distant organs [44]. Osteopontin is a secreted phosphoprotein which plays a critical role in metastasis of breast cancer. The canonical pathway for osteopontin signaling focuses on its binding interactions with integrin and CD44 cell surface receptors. However, the binding characteristic of osteopontin to integrin and CD44 receptors has not been previously examined and its research work is still going on. These additional receptors may play key functions in OPN's critical role in cancer metastasis [45].

\section{Stages of breast cancer}

Breast cancer staging using the TNM system is based on the size of the tumor (T), whether or not the tumor has spread to the lymph nodes $(\mathbf{N})$ in the armpits, and whether the tumor has metastasized (M) (i.e. spread to a more distant part of the body). Larger size, nodal spread, and metastasis have a larger stage number and a worse prognosis.

- Stage 0 is a pre-cancerous or marker condition, either ductal carcinoma in situ (DCIS) or lobular carcinoma in situ (LCIS).

- Stages 1-3 are within the breast or regional lymph nodes.

- Stage 4 is 'metastatic' cancer that has a less favorable prognosis.

Prostate cancer is considered as malignant tumor because it is a mass of cells that can invade other parts of the body. This invasion of other organs is called metastasis. Prostate cancer most commonly metastasizes to the bones, lymph nodes. It may also invade rectum, bladder and lower ureters after local progression. The route of metastasis to bone is thought to be venous as the prostatic venous plexus draining the prostate connects with the vertebral veins [46]. Interactions between prostate cancer cells and mature bone-producing osteoblasts resulted in gene expressional changes that may increase cells' ability to establish bone metastasis. The potential for inducing expressional changes seems to be crucial dependent of the differentiation status of osteoblasts [47].

Prostate cancer is staged to describe the amount and location of cancer. Staging systems also are used to determine treatment regimens. A "TNM" system is used to stage prostate cancer. It takes into consideration PSA level, tumor size, node involvement and amount of metastatic involvement. The " $\mathrm{T}$ " represents the tumor tissue size, the "N" represents the amount of lymph node involvement, and the " $\mathrm{M}$ " represents the extent to which the cancer has spread within the pelvic region (metastases). Each letter is combined with a number to explain the amount of variation among levels.

\section{Treatments}

There are different types of therapies and treatments related to breast and prostate cancers like surgery, radiotherapy, chemotherapy, gene therapy, hormone replacement therapy, oncogene therapy, radical prostatectomy, laparoscopy, minimally invasive treatment, tumor immunotherapy, genetic immunotherapy, radionuclide therapy, targeted cancer treatments, adjuvant therapy, immunotherapy, antiangiogenic chemotherapy, chemo radiation, conventional radiotherapy etc.

\section{Surgery}

Surgery involves the physical removal of the tumor, typically along with some of the surrounding tissue and frequently sentinels node biopsy.

Standard surgeries for breast cancer include:

- Mastectomy: Removal of the whole breast.

- Quadrantectomy: Removal of one quarter of the breast.

- Lumpectomy: Removal of a small part of the breast.

If the patient desires, then breast reconstruction surgery, a type of cosmetic surgery, may be performed to create an aesthetic appearance. In other cases, women use breast prostheses to simulate a breast under clothing, or choose a flat chest. Women should be informed that arm problems are expected but non-threatening squeal of initial surgical treatment for breast cancer [48].

Surgical treatment for prostate cancer includes radical prostatectomy. This means surgical removal of all or part of the prostate gland by an incision.

\section{Chemotherapy}

The use of preoperative neoadjuvant chemotherapy in the treatment of breast cancer has been evolving during the past two decades. The benefits of neoadjuvant chemotherapy are: 1) shrinking large cancer tumors so that they are small enough to be removed by breastconserving surgery instead of radical mastectomy; 2) reducing the chances of spread through the bloodstream, clearing the tiny metastatic lesion, and lowering the possibility of drug fast; 3) understanding the sensitivity of the tumor to chemotherapy and also providing evidence to post-surgery chemotherapy. In summary it was suggested that ${ }^{18}$ F-FDG imaging with hybrid PET/ CT provides a cost-effective method, which could be well accepted for the low-income population in developing countries, to monitor early tumor response after three cycles of neoadjuvant chemotherapy in stage II and III breast cancer by using the reduction of N/T ratio as predictor [49]. For aggressive tumors, the literature recommends CHOP type chemotherapy and mastectomy with lymph node resection, if needed, for management of PNHBL [50].

The first approved chemotherapy agents, mitoxantrone and estramustine (Emcyt), were utilized for prostate cancer patients to help control pain and to relieve symptoms associated with their metastatic disease. Because of the drugs' inherent side effects, doctors were reluctant to prescribe chemotherapy unless patients were experiencing problems from the cancer. The next phase of research for chemotherapy is to find ways to make Taxotere-based chemotherapy better. One such promising agent, bevacizumab (Avastin), appears to do just that. Avastin is a monoclonal antibody that targets the VEGF receptor; its main mechanism of action is via anti-angiogenesis. The role of chemotherapy is gaining acceptance for men with high risk or metastatic prostate cancer. Now a day's interferons are also being targeted for treatment of prostate and breast cancer. Interferons (IFNs) may target cancer cells both through their regulation of the immune response, effect on angiogenesis and through direct effect on cancer cells. Unconjugated interferon induced growth arrest [51].

Resistance to apoptosis leads to the progression of many solid cancers. Azurin, a potent anticancer redox protein secreted by Pseudomonas aeruginosa ( $P$. aeruginosa) species has been reported to have activity against breast cancer cell lines; $P$. aeruginosa MTCC 
(Microbial Type culture collection) 2453 was the strain that secreted the most azurin and showed remarkable apoptosis in breast carcinoma cells like T- 47D and ZR-75-1 [52]. Butyric acid (BA), an extracellular metabolite produced from periodontopathic bacteria result in progression of oral cancer such as oral squamous cell carcinoma (OSCC) [53]. Emerging prognostic factors such as Nottingham prognostic index (NPI) or triple-negative status might improve the models currently used by clinicians [54]. Intensity Modulated Radiation Therapy (IMRT) is widely accepted as an appropriate method to treat tumors at many different anatomic locations including lungs [55]. Chemokines and Chemokine receptors play a role in Prostate Cancer Development and Progression. Bone marrow aspiration along with trephine biopsy is essential for the diagnosis and management of multiple myeloma [56].

\section{Radiotherapy}

Radiotherapy is one of the main treatments for breast and prostate cancers. Although treatment with a combination of radiotherapy with chemotherapy, and/or surgery improves the prognosis of patients, no biomarkers can distinguish between the responses obtained with the combined therapies. Therefore studies are with patients treated with radiotherapy alone to evaluate surviving as a predictor for radiotherapy. SWOG trial S0102 showed significant activity of the combination of docetaxel and vinorelbine in HER2-negative metastatic breast cancer (MBC). The combination of trastuzumab, docetaxel, and vinorelbine is effective as first-line chemotherapy in HER2-positive MBC with minimal toxicity. Postoperative adjuvant chemoradiotherapy was recommended as the standard treatment for patients with rectal cancer because it reduces local recurrence. This paradigm shifted with the use of neoadjuvant chemoradiotherapy, which not only reduces local recurrence but also improves sphincter preservation and surgical outcomes [57].

Radiotherapy is a common treatment for prostate cancer, but failure is observed 30 to $40 \%$ of the time. It is more common in patients with abnormal $\mathrm{p}^{53}$. It is more common in patients with abnormal $\mathrm{p}^{53}$. The phytochemical diferuloylmethane (curcumin) a naturally occurring flavinoid derived from the rhizome of Curcuma longa, shows potential radiosensitizing effects. In the present study, the effect of curcumin and radiation on cell viability, apoptosis and clonogenic cell death was examined in LNCaP (wild type $\mathrm{p}^{53}$ ) and PC3 (mutant $\mathrm{p}^{53}$ ) prostate cancer cells. The cell viability and cell proliferation decreased in presence of both curcumin and radiation as compared to curcumin or radiation alone in both the cell lines. Regardless of $\mathrm{p}^{53}$ status, combination treatment with curcumin $(2.5$ to $10 \mu \mathrm{M})$ and radiation (2 Gy) had a synergistic effect on clonogenic cell death in both LNCaP and PC3 cells. Curcumin appears to radiosensitize prostate cancer cells and may be a possible adjuvant to radiotherapy in the treatment of cancer.Thus there is a Synergistic Effect between Curcumin (diferuloylmethane) and Radiation on Clonogenic Cell Death Independent of $\mathrm{p}^{53}$ in Prostate Cancer Cells [58].

Many viruses have been associated with human breast cancers, human papilloma virus (HPV) strains HPV16 and HPV18 in breast carcinoma shows different phenotypic changes. HPV16 and HPV18 significantly inhibited proliferation and adhesion of T-47D cells, although viability was not affected. Differential effects on proliferation were observed in MCF7 cells; HPV16 inhibited proliferation, while HPV18 stimulated proliferation. No measurable effects in adhesion or viability in MCF7 cells were observed. The phenotypic changes in T-47D and MCF7 cells were associated with changes in mRNA expression of caspase- $2,-3$ and -8 , but not $\mathrm{p}^{53}$ [59].

\section{Recent advances in treatment of breast and prostate cancer}

Recent advances in diagnosis and treatment of cancer have led to an increase in cancer survival and hence, there is a greater emphasis on quality beside quantity of survival. However little is known about the Quality Of Life of survivors [60]. Azurin, a potent anticancer redox protein secreted by Pseudomonas aeruginosa ( $P$. aeruginosa) species has been reported to have activity against breast cancer cell lines; $P$. aeruginosa MTCC (Microbial Type culture collection) 2453 was the strain that secreted the most azurin and showed remarkable apoptosis in breast carcinoma cells like T- 47D and ZR-75-1 [52]. Neutropenic enterocolitis (typhilitis) is a rare complication of chemotherapy-related neutropenia in cancer patients. A case report on 3 homogeneously treated patients presenting neutropenic enterocolitis following Docetaxel and Epirubicin chemotherapy for advanced breast cancer suggests that in neutropenic patients, attention should be paid even to vague abdominal discomfort. Urgent CT scan evaluation and early surgery appear to be of great importance [61].

A non-invasive multispectral imaging platform for monitoring spectral reflectance and fluorescence images from primary breast carcinoma and metastatic lymph nodes in preclinical rat model in vivo was reported. Translational potential of the imaging platform in intraoperative clinical setting is also under discussion [62]. A question is posed as to whether following the detection of circulating tumor cells (CTCs) at the single-cell level after adjuvant treatment may be useful and feasible in determining breast cancer recurrence after treatment, as evidenced in the arena of metastatic breast cancer, as an indicator determining the full response to treatment [63]. Landmark studies by Cristofanilli et al. [64] have demonstrated that CTCs detected after treatment in metastatic breast cancer can indicate early progression of disease and help clinician decide on changing in therapeutic regimens sooner rather than later when disease is detected on scans.

The inhibition of $\mathrm{p}^{53}$ can protect normal cells during genotoxic chemotherapy or radiation therapy. The side effects of genotoxic therapy for cancer are largely caused by $\mathrm{p}^{53}$-mediated apoptosis. The small molecule pifithrin- $\alpha$ can block $\mathrm{p}^{53}$-dependent transcriptional activity and protect mice from the lethal side effects associated with anticancer treatment [65]. Metastasis Associated 1(MTA1) Aids the AKT Pathway by Inhibiting Expression of a Key Regulator, PTEN the phosphatase and tensin analogue mutated on chromosome 10. PTEN is a tumour suppressor gene known to be mutated in several cancers [66]. Endothelial antigens that stimulate immune-mediated damage of tumor vessels represent possible targets for the development of antiangiogenic vaccines aimed at preventing the progression of solid tumors [67]. S-1, an oral anticancer drug, caused only mild toxicity and had highly effective antitumor activity. Furthermore, the RR of S-1 NAC might predict regional lymph node metastasis [68]. FIR inhibited the proliferation of HepG2 at non-thermal circumstances (at $25 \pm 0.5,37 \pm 0.5^{\circ} \mathrm{C}$ ). FIR will serve as a tool against diseases induced by HepG2 [69]. Efficacy of transcatheter arterial infusion (TAI) alone or combined with transcatheter arterial chemoembolization (TACE) on advanced hepatocellular carcinoma (HCC) [70].

Effective tailored therapies have been developed for patients with hormone receptor-positive or $\mathrm{HER}^{2+}$ disease, chemotherapy is the only modality of systemic therapy for patients with breast cancers lacking the expression of these markers (triple-negative breast cancers). Thus, the development of biologically informed systemic therapies and targeted therapies for triple-negative breast cancers is of paramount importance and may prove to be a challenging task, only achievable 
by understanding the complexity of this heterogeneous group of tumors. Although triple-negative breast cancers are reported to respond to neoadjuvant chemotherapy, survival of patients with such tumors is still poor and their management may therefore require a more aggressive alternative intervention. Therefore, research is being carried out for a reliable biomarker or a target that could be used to individualize both patient prognosis and therapy is essential for the prevention and cure of triple negative breast cancers [71]. In recent years, many studies have examined the relationship between breast cancer outcomes and vitamin D. Vitamin D also plays an important role in breast cancer symptom management. Based on the evidence of ongoing trials, vitamin D supplementation may eventually be used as a cardioprotective agent in breast cancer patients [72].

In case of Prostate cancer using prostate specific antigen (PSA) as an indicator for the presence of bony metastases rather than routine bone scans would have large economic savings given the population size. However, these recommendations are restricted only to symptomatic screening and cannot extrapolate to population-based screening program due to the sample selection of the study [73]. Multicellular Spheroid Culture (MCS) models have become a mainstream culture model for tumor biology and identification of anticancer resistance mechanisms as an alternative to the classical 2D culture models that poorly reflect the structural characteristics seen in vivo. A review has highlighted the characteristics of prostate cancer MCS $\left(\mathrm{p}^{27}\right.$ and PARP expression, and epigenetics), and underlined the tumor microenvironment as target for prostate cancer therapy. MCS culture models appear to contribute to the identification of cancer stem cells from the prostate. Further studies are needed to clarify mechanisms such as epigenetic regulation, to better characterize the formation of MCS, and to apply this knowledge into prostate cancer biology and the discovery of new targets for prostate cancer [74]. A nanoparticle based imaging agent that targets SPARC (Secreted Protein Acidic Rich in Cysteine), a molecular marker of prostate cancer metastatic potential has been developed. In vivo imaging information correlates with the metastatic potential of the prostate tumor. This prognostic information could enable doctors to stratify patients and design personalized treatment strategies [75].

A review article on prostate cancer provides information on 4 new potential treatments (cardiac hormones) of prostate cancer and their molecular targets (Ras-MEK 1/2-ERK 1/2 kinase cascade). Vessel dilator, long-acting natriuretic peptide, atrial natriuretic peptide and kaliuretic peptide each significantly decrease up to $97 \%$ of human prostate cancer cells in culture. After binding to specific receptors, their signaling in prostate cancer cells involves at 95\% inhibition of the activation of Ras, $98 \%$ inhibition of the activity of MEK $1 / 2$ kinases, and $96 \%$ inhibition of the phosphorylation of ERK $1 / 2$ kinases mediated by cyclic GMP. They also inhibit DNA synthesis within prostate cancer cells mediated by cyclic GMP [76]. Combinations of phytoestrogens with radiation therapy and other antioxidants in advanced or metastatic prostate cancer can be considered because there are limited effective therapy options for this group of patients [77]. A current review states that Prostate Cancer Prevention Trial [PCPT] and Reduction by Dutasteride of Prostate Cancer Events [REDUCE] trials reveal that 5-ARI's have the ability to reduce low grade prostate cancer, but also show a slight increase high grade prostate cancer diagnosis. Follow-up studies suggest that these findings may be due to detection bias, but official guidelines recommend against 5-ARI use due to the potential risk. Efficacy of chemoprevention agents enables: prevention of over-diagnosis, decreased use of overly aggressive treatment, and enhanced quality of life by reducing suffering, mortality, and economic burdens [78]. A comparative proteomic study of proteins in cultivated cells DU-145, PC-3, LNCaP and BPH-1, as well as in prostate tissue samples obtained from patients with malignant and benign tumors is being done [79].

Cancer treatment involving chemotherapy is typically accompanied by toxic side effects, thereby limiting the amount of the drug that can be given to a patient. As a result, all of the tumor tissue may not be exposed to a lethal dose of the drug. The use of nanocarriers such as liposomes and micelles can improve the pharmacological properties of traditional chemotherapeutics. Encapsulated drug is unable to undergo nanocarrier/endosomal escape. However, a combinatorial approach in which surface modifications made to nanocarriers involve both a targeting ligand as well as a $\mathrm{p}^{\mathrm{H}}$-triggered release mechanism following cellular internalization based on endosomal $\mathrm{pH}$ may in fact generate a very efficacious construct. This type of combinatorial approach in nanocarrier design may in fact prove to someday be a very efficacious drug delivery system commonly used in cancer therapy [80]. Pretreatment dose verification is carried out at almost all the centers but measurement techniques and plan acceptance criteria are institution specific. Thus, a variety of IMRT QA program in totality is being followed at the Indian hospitals. There is a need to evolve a national protocol for IMRT QA so that treatment outcomes of all the IMRT centers of country can be compared [81].

The cancer stem cell theory proposes that there is a small but constant subpopulation of cancer cells with stem cell properties responsible for the self renewal capacity and unlimited proliferation of tumor as well as increased resistance to antineoplastic drugs. Targeting these cells might constitute an effective way to cure cancer. All glioma cells have stem cells properties but their phenotype varies depending on the environmental conditions. This model provides an alternative explanation to different and sometimes controversial experimental findings and might be a useful guide for future research in the field of gliomas and stem cell biology [82]. The use of the $\mathrm{RC}_{0}$ has the potential to increase the successful rate and maybe, overcome the bottleneck when translating preclinical research into clinical trials. From the economical perspective, predicting which drug will successfully pass clinical trials will have a tremendous impact in the drug industry by lowering cost and time by stopping clinical trials of high number of drugs. On the other hand the use of the $\mathrm{RC}_{0}$ will help at early stages to decide which drugs have little chance to be useful for monotherapy but might have use in combinational regimes [83]. MU-Tomo can perform in a few seconds an independent dose calculation accurately and provide a secondary check for a point dose validation of helical tomotherapy plans [84]. To provide more clinically convenient image fusions for adaptive radiotherapy (ART), an automatic rigid and deformable image registration framework (AIRF) is developed for multimodal visualization of multiple chronological images and multiple radiotherapy (RT) plans. The AIRF can automatically register multiple volumetric image datasets of patients taken over an extended period of time and can merge multiple RT plans based on different planning CT images for 4D or adaptive radiotherapy [85]. Incorporation of a pertinent tumor endothelial biomarker into a drug development process could also reduce the time, size and cost of clinical trials through the selection of patients who are responding to novel therapies, as well as increasing the chances of regulatory approval of new leads. Finally, the capability to monitor patient response to a targeted treatment through the read out of the vascular integrity can be used to rescue drugs that may benefit specific populations but whose 
clinical efficacy was missed in unselected populations dominated by non-responders [86]. Any discussion on cancer biology, directly or indirectly, includes stem cells, in particular, tumor initiating cells and mesenchymal stem cells (MSCs). The question of targeting cancer stem cells and also the supporting MSCs in cancer cannot be achieved by one entity, but partnership among academia, biotechnology and pharmaceuticals [87]. The field of nanomedicine has proved a feasibility of active targeting using different types of ligand as well as nanoparticles. To this end, we emphasize a need of novel ligand beyond antibodies that targets different tumor component including those in the tumor microenvironment to achieve comprehensive cancer treatment [88].

\section{Conclusion}

This review gives an overall view on two most prevalent and hazardous cancers, the breast and prostate. It addresses the risk factors like genetical, hormonal, dietary etc. Relation between these cancers and metabolic syndrome is given. Mainly it covers all the recent advances in treatment of breast and prostate cancers. Further research in the treatment of these cancers can yield fruitful results.

\section{References}

1. Ahmedin Jemal DVM, Rebecca Siegel MPH, Elizabeth Ward, Yongping Hao, Jiaquan Xu MD, et al. (2009) Cancer Statistics, 2009. CA Cancer J Clin 59: 225-249.

2. Bareggi Renato NV, Paola N (2010) New Targeted Therapies Against Breast Cancer. J Carcinogene Mutagene 1:110.

3. Singh RK, Sudhakar A, Lokeshwar BL (2010) Role of Chemokines and Chemokine Receptors in Prostate Cancer Development and Progression. J Cancer Sci Ther 2: 094-099.

4. Risbridger GP, Davis ID, Birrell SN, Tilley WD. (2010) Breast and prostate cancer: more similar than different. Nat Rev Cancer 10: 205-212.

5. Pharoah PD, Day NE, Duffy S, Easton DF, Ponder BA. (1997) Family history and the risk of breast cancer: a systematic review and meta-analysis. Int $\mathrm{J}$ Cancer 71: 800-809.

6. Lesko SM, Rosenberg L, Shapiro S. (1996) Family History and Prostate Cancer Risk. Am J Epidemiol 144:1041-1047.

7. Germain D (2011) Estrogen carcinogenesis in breast cancer. Endocrinol Metab Clin North Am 40: 473-484

8. Jamil K, Kumar K, Fatima SH, Rabbani S, Kumar R, et al. (2009) Clinical Studies on Hormonal Status in Breast Cancer and its Impact on Quality of Life (QOL). J Cancer Sci Ther 1: 083-089.

9. Chan JM, Stampfer MJ, Giovannucci EL (1998) What causes prostate cancer? A brief summary of the epidemiology. Semin Cancer Biol 8: 263-273.

10. Taylor RA, Toivanen R, Risbridger GP (2010) Stem cells in prostate cancer: treating the root of the problem. Endocr Relat Cancer 17: R273-285.

11. Sahu B, Laakso M, Ovaska K, Mirtti T, Lundin J, et al.(2011) Dual role of FoxA1 in androgen receptor binding to chromatin, androgen signalling and prostate cancer. EMBO J 30: 3962-3976.

12. Holmes MD, Willett WC (2004) Does diet affect breast cancer risk? Breast Cancer Res. 6: 170-178.

13. Stajner I (2009) Cloudiness and Breast Cancer. J Cancer Sci Ther 1: 034-040.

14. Vasundara V, Laurence HK (2010) Diet and prostate cancer: mechanisms of action and implications for chemoprevention. Nat Rev Urol 7: 442-453.

15. Key TJ (2011) Fruit and vegetables and cancer risk. Br J Cancer 104: 6-11.

16. Alexander DD, Mink PJ, Cushing CA, Sceurman B (2010) A review and metaanalysis of prospective studies of red and processed meat intake and prostate cancer. Nutr J 9: 50
17. Donald TW, Michelle CT, James G, Marie-Élise P (2008) Role of Hormonal and Other Factors in Human Prostate Cancer. J Toxicol Environ Health 11: 242 259

18. Schulman CC, Ekane S, Zlotta AR (2001) Nutrition and prostate cancer evidence or suspicion? Urology 58: 318-334

19. John EM, Phipps Al, Knight JA, Milne RL, Dite GS, et al. Medical radiation exposure and breast cancer risk: findings from the Breast Cancer Family Registry. Int J Cancer 121: 386-394.

20. Jacobs EJ, Rodriguez C, Mondul AM, Connell CJ, Henley SJ, et al. (2005) A Large Cohort Study of Aspirin and Other Nonsteroidal Anti-inflammatory Drugs and Prostate Cancer Incidence. J. Natl Cancer Inst 97: 975-980.

21. Shannon J, Tewoderos S, Garzotto M, Beer TM, Derenick R, et al. (2005) Statins and Prostate Cancer Risk: A Case-Control Study. Am J Epidemiol 162 318-325.

22. Kingsley K, Zuckerman J, Davis M, Matteucci M, Knavel A, et al. (2009) Induction of Differential Growth in vitro by High-risk Human Papillomavirus in Human Breast Cancer Cell Lines is Associated with Caspase Dysregulation. J Cancer Sci Ther 1: 062-071.

23. Urisman A, Molinaro RJ, Fischer N, Plummer SJ, Casey G, et al. (2006) Identification of a Novel Gammaretrovirus in Prostate Tumors of Patients Homozygous for R462Q RNASEL Variant. PLoS Pathog 2: e25.

24. Schlaberg R, Choe DJ, Brown KR, Thaker HM, Singh IR (2009) XMRV is present in malignant prostatic epithelium and is associated with prostate cancer, especially high-grade tumors. Proc Natl Acad Sci 106: 16351-16356.

25. Raymond PB, Raymond SG, Whitaker JP (1986) Racial differences in survival of women with breast cancer. J Chronic Dis 39: 631-642.

26. Morton RA (1994) Racial differences in adenocarcinoma of the prostate in North American men. Urology 44: 637-645.

27. Ann WH, Lori CS, Streamson CC (2007) Obesity, metabolic syndrome, and prostate cancer. Am J Clin Nutr 86: 843S-857S.

28. Van der burg B (1991) Sex steroids and growth factors in mammary cancer. Acta Endocrinol 125: 28-41.

29. Benson EA, Holdaway IM (1982) Regulation of insulin binding to human mammary carcinoma. Cancer Res 42: 1137-1141.

30. Chisato N, Hiroyuki S, Rieko T,Hayashi M, Noriyuki T, et al. (2000) Relations of Insulin Resistance and Serum Concentrations of Estradiol and Sex Hormonebinding Globulin to Potential Breast Cancer Risk Factors. Cancer Science 91 948-953.

31. Stoll BA (2000) Adiposity as a risk determinant for postmenopausal breast cancer. Int J Obes Relat Metab Disord 24:527-533.

32. Sudhakar A (2009) Oral Submucous Fibrosis: Review on Etiopathogenesis. J Canc Sci Ther 1: i-iv.

33. Saslow D, Hannan J, Osuch J, Alciati MH, Baines C, et al. (2004) Clinica breast examination: practical recommendations for optimizing performance and reporting. CA Cancer J Clin. 54: 327-344.

34. Yu YH, Liang C, Yuan XZ (2010) Diagnostic value of vacuum-assisted breast biopsy for breast carcinoma: a meta-analysis and systematic review. Breast Cancer Res Treat 120: 469-479.

35. Samuel WB, William EG, Crowe DR, Michael GC, Heidi LW, et al. (2001) Molecular Biomarkers for Breast Cancer Prognosis: Coexpression of c-erbB-2 and $\mathrm{p}^{53}$. Ann Surg 233: 630-638.

36. Razmkhah M, Jaberipour M, Ghaderi A (2011) Chemokines and Chemokine Receptors Expression in the Adipose Derived Stem Cells (ASCs), Breast Tissues and in Peripheral Blood of Patients with Breast Cancer. J Carcinogene Mutagene 2:120.

37. Irving JB, Christine K, Paul MR, lan GR, Stephen GB, et al. (2000) Diagnosis of breast cancer using elastic-scattering spectroscopy: preliminary clinical results. J Biomed Opt 5: 221

38. Michael JB (2001) Prostate-Specific-Antigen Testing for Early Diagnosis of Prostate Cancer. N Engl J Med 344: 1373-1377. 
39. Essink-Bot ML, de Koning HJ, Nijs HG, Kirkels WJ, van der Maas PJ, et al. (1998) Short-term effects of population-based screening for prostate cancer on health-related quality of life. J Natl Cancer Inst 90: 925-31.

40. Sai YRKM, Dattatreya A, Anand SY, Mahalakshmi D (2011) Biomarkers and their Role in Premonition, Interpretation and Treatment of Cancer. J Cancer Sci Ther R1:002.

41. Cavalieri E, Chakravarti D, Guttenplan J, Elizabeth H, James I, et al. (2006) Catechol estrogen quinones as initiators of breast and other human cancers: Implications for biomarkers of susceptibility and cancer prevention. Biochim Biophys Acta 1766: 63-78

42. Bali A, Singh MP, Padmavathi, Khorate M, Ahmed J (2010) Malignant Fibrous Histiocytoma - An Unusual Transformation from Benign to Malignant. J Cancer Sci Ther 2: 053-057.

43. London SJ, Connolly JL, Schnitt SJ, Colditz GA. (1992) A Prospective Study of Benign Breast Disease and the Risk of Breast Cancer. JAMA 267: 941-944.

44. Dunning AM, Healey CS, Pharoah PD, Teare MD, Ponder BA, et al. (1999) A Systematic Review Of Genetic Polymorphisms and Breast Cancer Risk. Cancer Epidemiol Biomarkers Prev 8: 843-854.

45. Mi Z, Guo H, Markovic J, Kuo PC (2009) Characterization of Osteopontin Binding Kinetics In MDA-MB231 Breast and SK-Hep-1 Liver Cancer Cells. J Cancer Sci Ther 1: 047-051.

46. "Male Genitals - Prostate Neoplasms". Pathology study images. University of Virginia School of Medicine. Archived from the original on 2011-04-28. Retrieved 2011-04-28. "There are many connections between the prostatic venous plexus and the vertebral veins. The veins forming the prostatic plexus do not contain valves and it is thought that straining to urinate causes prostatic venous blood to flow in a reverse direction and enter the vertebral veins carrying malignant cells to the vertebral column."

47. Skogseth H, Dybwad M, Flatberg A, Halgunset J (2010) Mature BoneProducing Osteoblasts Alter Gene Expression of Metastasis Related Genes in Prostate Cancer Cells. J Carcinogene Mutagene 1:111.

48. Maunsell E, Brisson J, Deschênes L (1993) Arm problems and psychological distress after surgery for breast cancer. Can J Surg 36: 315-320.

49. Song WZ, Wang Z, Li LW, Chen JH, Wang J (2010) Evaluation of Early Response to Neoadjuvant Chemotherapy in Breast Cancer Patients by 18F-FDG and 99mTc-HL91 Imaging. J Nucl Med Radiat Ther 1:102.

50. Nigam A, Singh AK, Singh SK, Singh N, Singh N, et al. (2011) Primary Mammary (Non-Hodgkin) Lymphoma of Breast: A Case Report. J Cancer Sci Ther 3: 173-175.

51. Müller CR, Namløs HM, Bjerner J, Østensen IHG, Saeter G, et al. (2010) Characterization of Treatment Response to Recombinant Interferon- a2b in Osteosarcoma Xenografts. J Cancer Sci Ther 2: 033-042.

52. Ramachandran S, Sarkar S, Mazumadar A, Mandal M (2011) Azurin Synthesis from Pseudomonas Aeruginosa MTCC 2453, Properties, Induction of Reactive Oxygen Species, and p53 Stimulated Apoptosis in Breast Carcinoma Cells. J Cancer Sci Ther 3: 104-111.

53. Miyazaki Y, Kikuchi K, González-Alva P, Inoue H, Noguchi Y, et al. (2010) Association of Butyric Acid Produced by Periodontopathic Bacteria with Progression of Oral Cancer. J Cancer Sci Ther 2: 026-032.

54. Nieder C, Spanne O, Bilberg I, Dalhaug A (2010) Primary Tumour Characteristics as Potential Prognostic Factors in Brain Metastases from Breast Cancer. J Cancer Sci Ther 2: 4

55. Aljarrah K, Pawlicki T, Niemierko A, Mell LK, Jiang SB (2010) A Clinical Study of MLC-Based IMRT Lung Dose Calculation Accuracy on Plan Evaluation Parameters. J Cancer Sci Ther 2: 070-073.

56. Jayashankar E, Roshinipaul T (2010) Prognostication of Histomorphologica Characteristics in Multiple Myeloma. J Cancer Sci Ther 2: 153- 156.

57. Naga Deepthi CH, VVL Pavan Kumar A, Rameshbabu and U Indirapriyadarshini (2011) Role of Tumor Suppressor Protein p53 in Apoptosis and Cancer Therapy. J Cancer Sci Ther R1-001.

58. Nayak BK, Krishnegowda NK, Galindo CA, Meltz ML, Swanson GP (2010)
Synergistic Effect Between Curcumin (diferuloylmethane) and Radiation on Clonogenic Cell Death Independent of p53 in Prostate Cancer Cells. J Cancer Sci Ther 2: 171-181.

59. Tsimafeyeu I, Demidov L, Kharkevich G, Petenko N, Volkova M, et al. (2010) Granulocyte-Macrophage Colony-Stimulating Factor, Interferon Alpha and Interleukin-2 as Adjuvant Treatment for High-Risk Renal Cell Carcinoma. J Cancer Sci Ther 2: 157-159.

60. Pakseresht S, Ingle GK, Garg S (2011) Quality of Life of Women with Breas Cancer at the Time of Diagnosis in New Delhi. J Cancer Sci Ther 3: 066- 069.

61. Lombardi D, Venturini S, Veronesi A (2011) Neutropenic Enterocolitis as Possible Complication of Docetaxel and Epirubicin Chemotherapy for Breast Cancer: Report of 3 Cases. J Cancer Sci Ther 3: 186-187.

62. Ramanujan VK, Ren S, Park S, Farkas DL (2010) Non-invasive, Contrastenhanced Spectral Imaging of Breast Cancer Signatures in Preclinical Animal Models In vivo. J Cel Sci Therapy 1:102.

63. Morris GJ (2011) Circulating Tumor Cells in Breast Cancer: Are They Indicative of the State of Disease? J Cell Sci Ther 2:101e.

64. Cristofanilli M, Budd GT, Ellis MJ, Stopeck A, Matera J, et al. (2004) Circulating tumor cells, disease progression, and survival in metastatic breast cancer. The New Engl J Med 351: 781-791.

65. Kikawa KD, Noah T, Ahwah SM, Pardini RS (2011) Docosahexaenoic Acid (DHA) Induces P53-Dependent Growth Inhibition in Transformed Colon and Lung Cell Lines Expressing Wildtype P53. J Cancer Sci Ther 3: 4.

66. Shimul S, Rajiv G (2010) MTA1 Aids the AKT Pathway by Inhibiting Expression of a Key Regulator, PTEN. J Cancer Sci Ther 2: 114-119

67. Balashova EE, Lokhov PG (2010) Proteolytically-cleaved Fragments of Cell Surface Proteins Stimulate a Cytotoxic Immune Response against Tumoractivated Endothelial Cells In vitro. J Cancer Sci Ther 2: 126-131.

68. Yokoe H, Kasamatsu A, Ogawara K, Ishigami T, Sato Y, et al. (2010) Neoadjuvant Chemotherapy with S-1 for Patients with Oral Squamous Cel Carcinoma. J Cancer Sci Ther 2: 132-135.

69. Ishikawa T, Ishibashi J, Yamashita K, Dalkhsuren SO, Sumida K, et al. (2009) Non-thermal Effects of Far-Infrared Ray (FIR) on Human Hepatocellular Carcinoma Cells HepG2 and their Tumors. J Cancer Sci Ther 1: 078-082.

70. Hao M, Lin H, Chen Q, Yu W, Zhou D, et al. (2011) Efficacy of Transcathete Arterial Infusion Alone or Combined with Transcatheter Arteria Chemoembolization on Advanced Hepatocellular Carcinoma. J Cancer Sci Ther 3: 130-133.

71. Srivastava M, Eidelman O, Bubendorf L, Pollard HB (2011) ANXA7 Expression has Prognostic Impact for Patient Survival In Triple Negative Negative Breast Cancers. J Mol Biomark Diagn 2:110.

72. Janelsins MC, Mustian KM, Peppone LJ, Sprod LK, Shayne M, et al. (2011) Interventions to Alleviate Symptoms Related to Breast Cancer Treatments and Areas of Needed Research. J Cancer Sci Ther S2. [Ahead of print]

73. Abdellatif J, Hajji F, Elonodo JC, Ghadouane M, Ameur A, et al. (2011) Update the Indicator Role of Serum PSA Level and Gleason Score of the Biopsy for the Presence of Bony Metastases: Bone Scan Findings in a North African Ethnic Group. J Cancer Sci Ther 3: 112-115.

74. Kurioka D, Takagi A, Yoneda M, Hirokawa Y, Shiraishi T, et al. (2011) Multicellular Spheroid Culture Models: Applications in prostate Cancer Research and Therapeutics. J Cancer Sci Ther 3: 060-065.

75. Thomas S, Waterman P, Chen S, Marinelli B, Seaman M, et al. (2011) Development of Secreted Protein and Acidic and Rich in Cysteine (SPARC) Targeted Nanoparticles for the Prognostic Molecular Imaging of Metastatic Prostate Cancer. J Nanomedic Nanotechnol 2:112.

76. Vesely DL (2011) Cardiac Hormones for the Treatment of Prostate Cancer. J Cancer Sci Ther S1. [Ahead of print]

77. Kolukula S, Anderson RJ (2011) Phytoestrogens and their Potential Role in Prostate Cancer Prevention and Treatment. J Cancer Sci Ther S1. [Ahead of print]. 
Citation: Shalini G, Phaneendra M, Anamika K, Jyothi J (2011) An Eye on Breast and Prostate Cancers. J Cancer Sci Ther S17. doi:10.4172/19485956.S17-003

Page 9 of 9

78. Correll Abbey BS, Barqawi AI MD (2011) Prostate Cancer Chemoprevention: A Current Review. J Cancer Sci Ther S3:002. [Ahead of print]

79. Sergey S, Marina K, Alexei I, Lidia E, Ksenia L (2011) Comparative Proteomic Study of Proteins in Prostate Cancer and Benign Hyperplasia Cells. J Cancer Sci Ther S1. [Ahead of print]

80. Khan DR (2010) The use of Nanocarriers for Drug Delivery in Cancer Therapy. J Cancer Sci Ther 2: 058-062.

81. Kumar R, Sharma SD, Amols HI, Mayya YS, Kushwaha HS (2010) A Survey on the Quality Assurance Procedures Used in Intensity Modulated Radiation Therapy (IMRT) at Indian Hospitals. J Cancer Sci Ther 2: 166-170.

82. Cruz M, Siden Å, Tasat DR, Yakisich JS (2010) Are all Glioma Cells Cancer Stem Cells? J Cancer Sci Ther 2: 100-106.

83. Avramidis D, Cruz M, Sidén Å, Tasat DR, Yakisich JS (2009) Regrowth Concentration Zero (RC0) as Complementary Endpoint Parameter to Evaluate Compound Candidates During Preclinical Drug Development for Cancer Treatment. J Cancer Sci Ther 1: 019-024.
84. Wang CY, Lee TF, Fang CH (2009) 3D Volumetric Visualization with Automatic Rigid and Deformable Hybrid Image Registration for Adaptive Radiotherapy. J Cancer Sci Ther 1: 041-000.

85. Papanikolaou N, He W, Vazquez LA, Gutierrez A, Stathakis S, et al. (2010) MU-Tomo: Independent Dose Validation Software for Helical TomoTherapy. J Cancer Sci Ther 2: 145-152.

86. Sarrazin S, Maurage CA, Delmas D, Lassalle P, Delehedde M (2010) Endocan as a Biomarker of Endothelial Dysfunction in Cancer. J Cancer Sci Ther 2: 047052.

87. Rameshwar P (2011) Post-identification of Cancer Stem Cell: Ethical and Scientific Dilemmas in Therapeutic Development? J Stem Cell Res Ther 1:e101.

88. Suh KS, Tanaka T (2011) Nanomedicine in Cancer. Translational Medic $1: 103 e$. 